\title{
Leitura compartilhada de histórias e aprendizagem de palauras em crianças típicas e com Síndrome de Down
}

\author{
Lectura compartida de historias y aprendizaje de palabras en niños \\ con desarrollo típico y con Síndrome de Down \\ Shared Book Reading and Word Learning in Children with \\ Typical Development and with Down Syndrome
}

\author{
Camila Bonagamba* \\ Andréia Schmidt* \\ Universidade de São Paulo e Instituto Nacional de Ciência e Tecnologia \\ sobre Comportamento, Cognição e Ensino
}

Doi: http://dx.doi.org/10.12804/revistas.urosario.edu.co/apl/a.5975

\section{Resumo}

\begin{abstract}
A leitura compartilhada de histórias é um recurso para ensino de vocabulário para crianças pré-escolares, porém não se sabe sobre a sua eficácia para crianças com Síndrome de Down (SD). Este estudo investigou a aprendizagem de relações entre palavras (um substantivo e um adjetivo) e figuras, por crianças com SD e com desenvolvimento típico (DT), a partir da leitura repetida de uma história. Seis crianças DT (3-4 anos) e seis SD (5-8 anos) participaram de três sessões indivi-
\end{abstract}

duais de leitura de um livro não comercial, que continha duas palavras desconhecidas e suas respectivas figuras. Cada sessão tinha uma condição diferente de leitura. Antes do procedimento e ao final das sessões foram realizadas sondas de nomeação, emparelhamento com o modelo e exclusão. Uma semana após a última sessão as sondas foram reaplicadas, acrescidas de sondas de generalização. As crianças DT demostraram aprendizagem da relação palavra-fruta nas sondas. Dois participantes SD demonstraram indícios de aprendizagem da relação palavra-fruta, mas não da relação palavra-cor.

* Universidade de São Paulo e Instituto Nacional de Ciência e Tecnologia sobre Comportamento, Cognição e Ensino Nota das autoras

Essa pesquisa faz parte do programa científico do Instituto Nacional de Ciência e Tecnologia sobre Comportamento, Cognição e Ensino (INCT-ECCE), financiado pelo Conselho Nacional de Pesquisa (CNPq, Processo 465686/2014-1) e pela Fundação de Amparo à Pesquisa do Estado de São Paulo (FAPESP, Processo 2014/50909-8). A pesquisa também contou com o apoio da FAPESP, com auxílio regular à pesquisa para a segunda autora (processo 2014/22990-5).

Correspondências relacionadas a este artigo devem ser enviadas para Andréia Schmidt, Faculdade de Filosofia, Ciências e Letras de Ribeirão Preto (USP - FFCLRP), Departamento de Psicologia, Av. Bandeirantes, 3900, Bairro Monte Alegre, Ribeirão Preto, São Paulo, Brazil. CEP: 14040-901.

Correio electrônico: aschmidt@ffclrp.usp.br

Para citar este artigo: Bonagamba, C., \& Schmidt A. (2019). Leitura compartilhada de histórias e aprendizagem de palavras em crianças típicas e com Síndrome de Down. Avances en Psicología Latinoamericana, 37(1), 73-88. Doi: http://dx.doi. org/10.12804/revistas.urosario.edu.co/apl/a.5975 
O procedimento foi adequado para ensinar substantivos para crianças DT, porém não para crianças SD, que demandam procedimentos adicionais.

Palavras-chave: leitura compartilhada de livros, aprendizagem de palavras, Síndrome de Down.

\section{Resumen}

La lectura compartida de historias es un recurso en la enseñanza de vocabulario en niños, pero no se sabe si ese recurso es eficaz para niños con Síndrome de Down (SD). Este estudio investigó el aprendizaje de relaciones entre palabras (sustantivo y adjetivo) y figuras, en niños con SD y con desarrollo típico (DT) a partir de la lectura repetida de una historia. Seis niños DT (3-4 años) y seis SD (5-8 años) participaron en tres sesiones individuales de lectura de un libro que contenía dos palabras desconocidas, con sus respectivas figuras. Antes del procedimiento y al final de cada sesión fueron realizados sondeos de nombramiento, igualación de la muestra y exclusión; después de una semana de la última sesión, los sondeos fueron replicados, más sondeos de generalización. Los niños DT demostraron aprendizaje de la relación palabra-fruta en todos los sondeos. Dos participantes SD demostraron indicios de aprendizaje de la relación palabra-fruta, pero no de la relación palabra-color. El procedimiento demostró ser adecuado para enseñar sustantivos a niños DT, pero no para niños SD.

Palabras clave: lectura compartida de libros, aprendizaje de palavras, Síndrome de Down.

\section{fibstract}

Shared book reading is a resource for teaching vocabulary to preschool children. However, it is not known whether this resource is effective among children with Down Syndrome (DS). This study focused on the learning of relations between words (a noun and an adjective) and figures by both, children with DS and children with typical development (TD), from the repeated reading of a story. Six children with TD (3-4 years) and six DS (5-8 years) participated in three shared book-reading sessions, conducted individually. The book contained two unknown words and their respective figures. In each session, a different condition of reading was presented. Before the procedure and at the end of each session, probes of naming, matching to sample and exclusion responding were performed. One week after the last session, the probes were reapplied, plus generalization probes. TD children showed learning of the word-fruit relation in the probes. Two DS participants showed evidence of learning the word-fruit relation, but not the word-color relation. The procedure was adequate for teaching nouns for TD children with, but not for DS children, who require additional procedures.

Keywords: Shared book reading, learning of words, Down syndrome.

\section{Introdução}

A aprendizagem de novas palavras por crianças ao longo do seu desenvolvimento depende de uma série de condições, como a regularidade com a qual ela entrará em contato com essas palavras e a diversidade de contextos na qual aparecerão (Greer \& Longano, 2010; Horst, 2013). Além disso, também é importante o tipo de resposta que a criança emite em relação à própria palavra ou aos aspectos do mundo aos quais ela se refere, como olhar em direção ao aspecto referido pela palavra, repetir a palavra nova ou emitir uma ação diante da palavra - os chamados comportamentos de falante e de ouvinte (Skinner, 1957). No entanto, as palavras não são aprendidas de forma equivalente ao longo do processo, principalmente quando são de classes gramaticais diferentes (e.g., substantivos e adjetivos) (Kucker, Murray \& Samuelson, 2015; Schmidt, Costa, Norberto \& Voss, 2014).

Pesquisas sobre desenvolvimento indicam que substantivos (especialmente os concretos) são aprendidos com maior facilidade que outras categorias lexicais, como verbos e adjetivos, por exemplo (Booth \& Waxman, 2009; Tribushinina et al., 2013), o que tem sido referido como "hipótese 
do viés nominal" (Gentner, 1982; mas, ver Schmidt, et al., 2014; Tardif, Gelman \& Xu, 1999, para dados discordantes). Tal fato pode ocorrer porque o tipo de controle de estímulos requerido na aprendizagem de substantivos difere daquele requerido para propriedades de objetos (Waxman \& Booth, 2001): enquanto em substantivos concretos a relação se estabelece entre a palavra e o objeto como um todo, no caso de adjetivos a relação deve se estabelecer entre a palavra e propriedades específicas do objeto. Esse controle pode demandar uma exposição maior da criança à relação entre a palavra e a propriedade do objeto, ou dicas explícitas sobre o aspecto crítico do objeto relacionado à palavra nova. Além disso, adjetivos são menos frequentes na fala dos adultos (Tribushinina \& Gillis, 2012) e, para identificá-los corretamente, a criança deve ficar sob controle de dicas específicas em frases ou sentenças que ajudem a restringir o significado da palavra nova para uma única característica de determinado objeto, sendo necessário para isso o conhecimento prévio de outras classes gramaticais, como os substantivos (Booth \& Waxman, 2009).

Um contexto natural e incidental bastante comum (e eficiente) de aprendizagem/ampliação de repertórios verbais para crianças é a leitura de livros de histórias feita por adultos (e. g. Horst, Parsons \& Bryan, 2011; McLeod \& McDade, 2011; Sim \& Berthelsen, 2014; Walsh \& Blewitt, 2006; Williams \& Horst, 2014). De modo geral, o que as pesquisas sobre o tema têm verificado é que, no contexto de leitura de histórias, as variáveis importantes para a aprendizagem de novas palavras são as mesmas presentes em outras interações naturais entre a criança e o adulto. Exemplos dessas variáveis são o número de exposições das palavras desconhecidas ao longo da leitura e a repetição do contexto em que a palavra aparece (e.g., Horst et al., 2011), a realização de perguntas, pelo adulto, sobre a história ou as palavras desconhecidas (e.g., Hargrave \& Sénéchal, 2000; Sim \& Berthelsen, 2014; Walsh \& Blewitt, 2006) - o que tem sido chamado de leitura dialógica por diversos autores
(Chow, McBride-Chang, Cheung \& Chow, 2008; Sim \& Berthelsen, 2014; Rogoski, Flores, Gauche, Coêlho \& Souza, 2017). Além disso, o tipo de interação realizado com a criança durante a leitura, como a solicitação da nomeação de figuras com a palavra-alvo (também chamada de tato intraverbal - Greer \& Du, 2015; Greer \& Ross, 2008), e perguntas gerais sobre a palavra-alvo ou a sua repetição (Walsh \& Blewitt, 2006) também parecem ser condições importantes.

O contexto de leitura de histórias é especialmente relevante para a aprendizagem de vocabulário por crianças porque, além de ser uma atividade naturalmente reforçadora, pode apresentar diversas características que favorecem a aprendizagem de novas palavras, como dicas contextuais sobre o significado de novas palavras, a possibilidade de repetições das palavras novas em diferentes contextos da história e ilustrações que possibilitam à criança relacionar palavras desconhecidas a elementos presentes nas figuras. Pesquisas mostram que crianças pré-escolares passam a maior parte do tempo olhando para as ilustrações do livro em um episódio de leitura compartilhada e que sua inspeção à ilustração, em geral, corresponde aos elementos que aparecem na história (Evans \& Saint-Aubin, 2013). Além disso, a atenção às ilustrações de um livro durante sua leitura está positivamente correlacionada com a aprendizagem de palavras contidas na história e à compreensão do enredo (Kaefer, Pinkham \& Neuman, 2017). Nesse sentido, se as ilustrações de um livro claramente apresentam figuras relacionadas às palavras que aparecem na história, a aprendizagem de novas palavras é facilitada (Evans \& Saint-Aubin, 2013).

Uma forma de aumentar a discriminabilidade de um elemento desconhecido no contexto de uma ilustração pode ser apresentá-lo junto a outros elementos já conhecidos. Esse tipo de configuração favorece o chamado "responder por exclusão" (Dixon, 1977): o adulto, ao longo da história, pode ler uma palavra desconhecida da criança (e.g., hipopótamo); ao ouvi-la, a criança pode 
relacioná-la a um elemento desconhecido presente na ilustração do livro, quando este elemento aparece junto a outros conhecidos (e.g., a ilustração pode apresentar a figura de um leão, um elefante - conhecidos da criança - e um hipopótamo). Dessa forma, a criança relacionaria os estímulos desconhecidos (a palavra e a figura do hipopótamo) por excluir as figuras que já aprendeu a relacionar a alguns nomes (leão e elefante). Nesse sentido, um livro de história organizado especificamente para fornecer dicas explícitas sobre relações entre palavras e seus referentes pode ser um instrumento importante para o ensino de novas palavras, tanto para crianças com desenvolvimento típico como para crianças com dificuldades de linguagem, como é o caso de crianças com Síndrome de Down.

A Síndrome de Down (SD) é a causa genética mais comum de deficiência intelectual e está associada a dificuldades específicas na linguagem (Freire, Duarte \& Hazin, 2012). Algumas pesquisas têm sido realizadas com crianças com SD utilizando a leitura compartilhada de histórias, principalmente para a melhoria de consciência fonológica e de habilidades básicas de leitura (e.g., Burgoyne et al., 2012; van Bysterveldt, Gillon \& Moran, 2006), porém, os resultados positivos encontrados por pesquisas com leitura compartilhada de histórias na melhoria do vocabulário de crianças com desenvolvimento típico sugerem que essa pode ser uma prática efetiva também para crianças com SD.

O presente estudo teve por objetivo investigar a aprendizagem de relações entre palavras e figuras (um substantivo - nome de uma fruta; e um adjetivo - nome de uma cor) por crianças com SD e com desenvolvimento típico (DT), a partir da leitura repetida de uma história, que era realizada de três formas: (a) leitura do livro sem intervenção, (b) leitura do livro com solicitação de repetição da palavra-alvo (emissão de ecoico), e (c) leitura com perguntas para a criança sobre as palavras-alvo. Para isso, uma história não comercial e as suas respectivas ilustrações (fotos) foram criadas especialmente para a pesquisa, de tal forma que os elementos desconhecidos (a foto da fruta e a cor) fossem apresentados em contextos que favorecessem sua relação com a palavra desconhecida, ou seja, junto a figuras de mesma natureza (outras frutas e cores) e já conhecidos pela criança.

\section{Método}

\section{Participantes}

Participaram do estudo seis crianças com DT, com idades entre 3 e 4 anos (nível de vocabulário de 4 anos), e seis crianças com SD, com idades entre 5 e 8 anos e nível de vocabulário de 3 anos. Nenhuma das crianças sabia ler. As crianças foram recrutadas em suas escolas (ensino regular para as crianças com DT e ensino especial, para as crianças com SD) e constituíram uma amostra de conveniência. $\mathrm{O}$ vocabulário das crianças foi avaliado pelo teste ABFW (Andrade, Befi- Lopes, Fernandes \& Wertzner, 2004). Os pais foram informados sobre a pesquisa e assinaram um Termo de Consentimento Livre e Esclarecido autorizando a participação de seus filhos. Os procedimentos foram realizados nas dependências das escolas em que os participantes estudavam, em uma cidade do interior do Estado de São Paulo (Brasil). A pesquisa foi aprovada pelo Comitê de Ética em Pesquisa com Seres Humanos da Faculdade de Filosofia, Ciências e Letras de Ribeirão Preto - USP (CAAE $\left.\mathrm{n}^{\mathrm{o}} 24137013.1 .0000 .5407\right)$.

\section{Estímulos}

Os estímulos-alvo foram a figura de uma fruta desconhecida das crianças e seu respectivo nome (estímulo S1 - camapu) e a cor "bege", que aparecia em uma cesta de madeira (estímulo A1) nas fotos que ilustravam o livro. Nos testes, também era apresentado um quadrado pintado na cor bege (A1') (ver Figura 1 - a, b e c). 


\section{Material}

Foi construído um conjunto de seis fichas $(29,7$ x 21,0 cm), cada uma com seis figuras de dimensões iguais. As fichas continham fotos de frutas familiares (Ficha 1), fotos de frutas desconhecidas (e.g., lichia e umbu - Fichas 2 e 3), quadrados coloridos com cores conhecidas (Ficha 4) e quadrados coloridos com cores incomuns (e.g., oliva, magenta, bege - Fichas 5 e 6 ).

Também foi produzido um livro de história infantil (ver Figura 1 - painéis “ $d$ " e "e"), contendo 15 páginas, com uma história apropriada para a idade das crianças. As ilustrações do livro eram fotografias produzidas pelas pesquisadoras a partir de cenas cotidianas e condizentes com o enredo da história. Todas as páginas do livro continham uma foto, que ocupava aproximadamente dois terços da página, e uma parte escrita, composta por frases curtas e que ocupavam aproximadamente um terço da página. Cada palavra-alvo e a respectiva figura (ou cor) apareceram no livro três vezes. As duas palavras-alvo (e suas respetivas figuras) nunca foram apresentadas juntas na mesma página. Nas fotos do livro, as figuras-alvo (S1 e A1) eram apresentadas duas vezes entre dois objetos conhecidos (ou cores, no caso de A1) e uma vez junto com um único objeto conhecido (ou cor conhecida).

Para as sondas de aprendizagem foram produzidas 18 fichas plastificadas $(29,7 \times 21,0 \mathrm{~cm})$; cada ficha correspondia a uma tentativa de teste. Seis fichas apresentavam uma única figura no centro da página (os três estímulos-alvo, mais duas figuras de frutas conhecidas - maçã e banana; e uma cor conhecida - preto) e foram empregadas em sondas de nomeação. As demais fichas apresentavam, cada

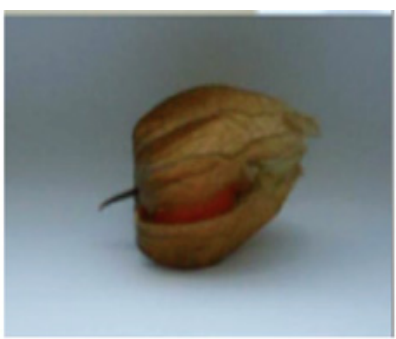

a

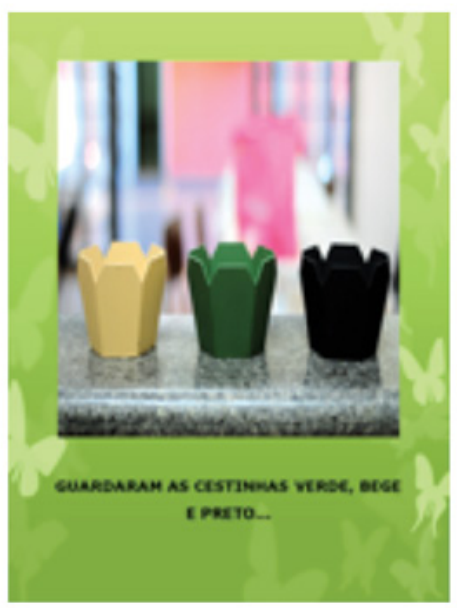

d

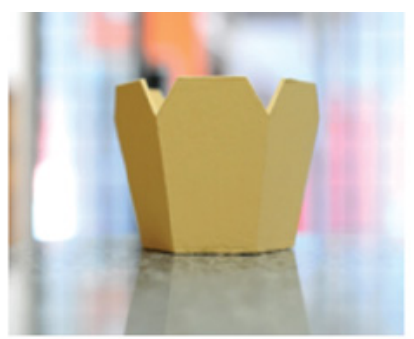

b

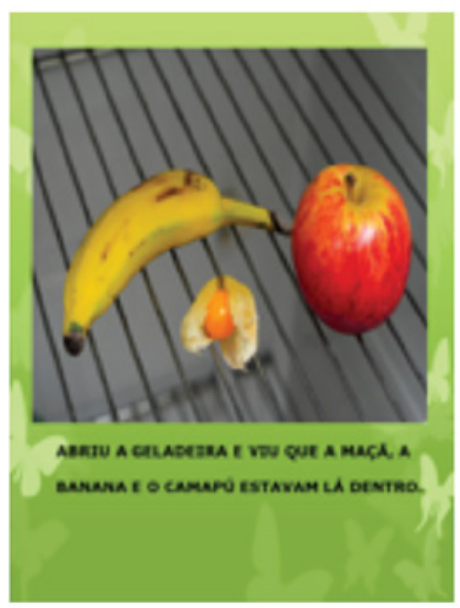

e

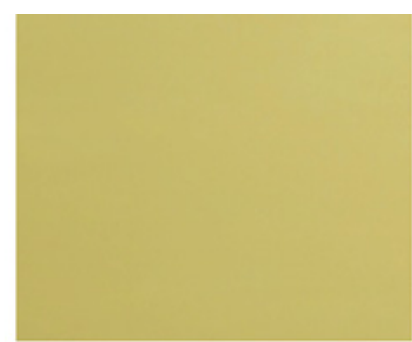

C

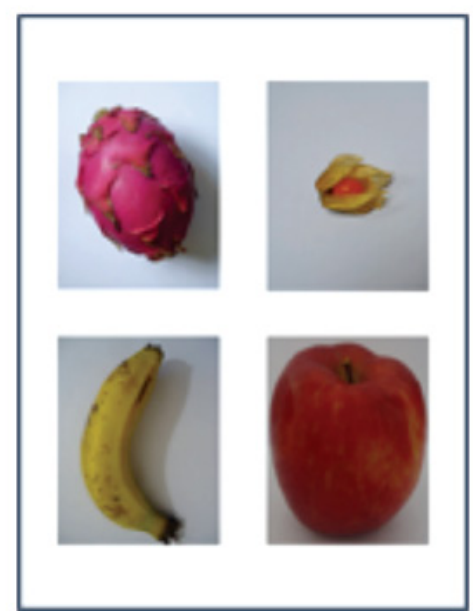

f

Figura 1. Exemplos dos estímulos empregados no estudo e a forma como foram apresentados: (a) S1 (camapu); (b) e (c) A1 e A1' (bege); (d) e (e) exemplos de páginas do livro de histórias; e (f) exemplo de página do livro de testes. 
uma, quatro figuras de mesma dimensão, dispostas nos quatro quadrantes da ficha (Figura 1 - painel “f”). Para os testes de generalização foram utilizadas frutas reais (entre elas o camapu), cestas coloridas e blocos de madeira coloridos (entre as cores, o bege).

Também foram utilizados adesivos coloridos $(5 \times 2 \mathrm{~cm})$, que a criança colava sobre a figura escolhida em resposta às tentativas de sonda.

\section{Procedimento}

A coleta de dados ocorreu nas escolas em que os participantes estudavam, em sala cedida pela instituição. Foi utilizado um delineamento de medidas repetidas, uma vez que todos os participantes passavam por todas as condições de leitura, com sondagens de aprendizagem após a condução de cada condição de leitura. O procedimento foi composto por quatro fases. Cada participante passava inicialmente por um pré-teste, para aferir seu conhecimento sobre os estímulos desconhecidos e os familiares, e depois passava por sessões semanais de leitura, nas quais ouvia a leitura do livro. Em cada sessão, após a leitura foram conduzidas as sondas de aprendizagem e, após uma semana da última sessão de leitura, uma última sondagem de manutenção da aprendizagem foi realizada.

Fase 1 - Pré-teste. O objetivo dessa fase foi verificar se as crianças relacionariam as palavras-ditadas aos os estímulos (frutas e cores) que seriam utilizados no livro e nos testes como estímulos-alvo e como estímulos conhecidos. As seis fichas foram apresentadas uma a uma para cada criança (ver Materiais). A cada ficha apresentada, a pesquisadora ditava uma palavra (o nome de uma das frutas ou das cores), e a criança deveria selecionar a figura que considerasse correta. A ficha era colocada no chão, em frente à criança e a pesquisadora entregava para a criança um adesivo. Em seguida, dizia, olhando em direção à criança (nunca em direção à ficha, evitando, assim, dar qualquer dica em relação à resposta correta): "Cole esse adesivo em cima do (e.g., maçã, ou camapú)?”; a pesquisadora olhava para a ficha somente depois da criança colar o adesivo sobre a figura que julgava ser a correta (registro da resposta). Nenhuma resposta, certa ou errada, era consequenciada diferencialmente, e logo após a resposta da criança a pesquisadora dizia: "Vamos ver o que tem na próxima?". Foram realizadas seis tentativas (uma para cada ficha), com as seguintes palavras ditadas: morango, camapú (duas vezes), preto e bege (duas vezes) (a ordem de apresentação das palavras-modelo/fichas foi sorteada). Essa fase se encerrava com a apresentação da última ficha. As respostas da criança ficavam registradas na própria ficha e eram anotadas pela pesquisadora ao final. $O$ critério para que a criança passasse à fase seguinte era ela selecionar incorretamente todos os estímulos desconhecidos e selecionar corretamente todos os conhecidos.

Fase 2 - Leitura compartilhada do livro. Nessa fase, foram conduzidas três sessões de leitura com cada criança individualmente e, em cada uma delas, era apresentada uma de três condições de leitura. O intervalo entre as sessões foi de aproximadamente uma semana. Em cada sessão, o livro era lido duas vezes em sequência (ao final da primeira leitura, a pesquisadora dizia "Vamos ler de novo?" e reiniciava a leitura). Durante a leitura do livro, a pesquisadora sentava-se no chão, ao lado da criança, com o livro colocado no chão, em frente a ambas, de modo que tanto a criança quanto a pesquisadora pudessem ver as fotos do livro. Durante a leitura, a pesquisadora não fazia nenhum gesto em direção às fotos do livro, permitindo, porém, que a criança explorasse a foto da maneira que quisesse. A ordem de apresentação das diferentes condições foi contrabalanceada entre os participantes, para controlar o efeito de ordem.

Condição 1-Somente leitura. Nessa condição, o livro foi lido duas vezes em sequência. A pesquisadora não fazia comentários sobre a história nessa 
condição. Se a criança fizesse alguma pergunta durante a leitura, a pesquisadora sugeria que continuassem com a leitura para ver o que acontecia (e.g., "Por que a sacola está mexendo?", ao que a pesquisadora respondia, olhando em direção à criança “Vamos continuar lendo para ver o que acontece?").

Condição 2 - Leitura com ecoico. Durante cada uma das duas leituras, na primeira vez em que as palavras-alvo (camapu e bege) apareciam na história, a pesquisadora pedia para a criança, “Vamos falar esse nome?". A pesquisadora, então, olhando em direção à criança, pronunciava pausadamente a palavra-alvo e dizia: "Agora é a sua vez de falar". Cada criança repetiu, portanto, duas vezes cada palavra-alvo, uma vez a cada leitura. Caso a criança não quisesse repetir alguma das palavras, a leitura continuava; caso ela errasse a pronúncia da palavra, não era corrigida e a leitura continuava, o mesmo ocorrendo nos casos em que a criança repetia corretamente a palavra. O procedimento descrito na Condição 1 era realizado caso a criança fizesse alguma pergunta.

Condição 3 - Leitura com perguntas. Durante cada leitura foram realizadas perguntas para a criança, cujas respostas eram as palavras-alvo presentes no livro. As respostas não foram consequenciadas diferencialmente. As perguntas foram realizadas na primeira vez em que cada palavra-alvo apareceu (portanto, a criança respondeu duas perguntas sobre cada palavra-alvo, uma em cada leitura). As perguntas eram: "Qual é a fruta que está em cima da mesa do lado da banana e da maçã?", e "Qual é a cor da cesta do lado da azul e da vermelha?".

Fase 3 - Sondas de aprendizagem. O objetivo dessa fase, que ocorreu após a apresentação de cada condição da Fase 2, foi verificar/monitorar a aprendizagem das palavras-alvo que eram apresentadas na história. Foram utilizadas as 18 fichas de teste nessa fase (ver seção "Material"), uma para cada tentativa. Em cada sondagem foram conduzidas: (a) seis tentativas de nomeação, sendo que em cada uma era apresentada uma única figura no centro da ficha e eram perguntados os nomes de estímulos conhecidos e dos estímulos-alvo (e.g., "Qual o nome dessa cor?", ou "Qual o nome dessa fruta?", para as figuras: banana, camapu, uma cesta de cor azul, a cor bege em um quadrado, a cor azul em um quadrado e a cesta de cor bege); (b) três tentativas de compreensão da tarefa, em que nomes de frutas conhecidas eram ditados e a criança deveria escolher entre quatro figuras de frutas/cores conhecidas apresentadas na ficha; (c) seis tentativas de emparelhamento ao modelo, em que cada estímulo-alvo (S1, A1 e A1') era ditado por duas vezes não consecutivas e as alternativas eram o estímulo-alvo e outros três estímulos desconhecidos (cores ou frutas) - a localização do estímulo correto foi balanceado entre as posições na página; (d) três tentativas de exclusão, na qual um nome completamente novo era ditado (que não aparecia na história e desconhecido da criança, como "Qual desses é o umbu?") e a criança tinha quatro opções de escolha - o estímulo-alvo, um estímulo completamente novo e dois estímulos conhecidos. Todas as sessões de testes foram filmadas para posterior análise e cálculo do índice de concordância.

Fase 4 - Sondas de Manutenção. Após uma semana da última condição de leitura, as sondas de aprendizagem (exclusão, emparelhamento ao modelo e nomeação) foram reaplicadas, a fim de avaliar se a aprendizagem das palavras se manteve. Além dessas sondas, foram realizadas seis tentativas de generalização, nas quais foram utilizadas frutas reais, cestas de diferentes cores (iguais às das fotos que ilustravam o livro) e blocos de madeira coloridos. Em cada tentativa de sonda de generalização o estímulo-alvo (a fruta, a cesta ou o quadrado colorido) era colocado sobre uma mesa, em frente à criança, entre outros três estímulos de mesma categoria (ou frutas desconhecidas, cestas 
ou blocos de madeira pintados com cores desconhecidas). Foi apresentada uma tentativa para cada estímulo-alvo e três tentativas com estímulos conhecidos (uma com frutas e duas com cores).

A duração de cada sessão, em todas as fases, foi, em média, de 11 minutos para as crianças com SD e de 6 minutos para as participantes com DT.

\section{Procedimento de análise de dados}

Os dados obtidos foram analisados quantitativamente, levantando a frequência de respostas corretas de cada grupo no pré-teste e em cada sondagem. Duas pesquisadoras com formação em Psicologia e experiência em análise do comportamento analisaram $100 \%$ das filmagens das sondas de nomeação. A concordância foi de 96,5\% para as nomeações realizadas pelas participantes com SD e de $95,8 \%$ para os participantes com DT.

\section{Resultados}

A Figura 2 apresenta a frequência de acertos dos grupos com DT e SD nas sondas de exclusão, emparelhamento ao modelo e nomeação no pré-teste (exceto na nomeação), em cada sondagem pós-leitura (T) e na manutenção (Man). O número máximo de acertos dos grupos a cada sondagem considerava apenas as tentativas que envolviam os estímulos-alvo ( $\mathrm{S} 1, \mathrm{~A} 1$ e A1'), e variou entre os desempenhos avaliados: por exemplo, o número máximo de acertos por grupo nas sondas de emparelhamento ao modelo era 12 , enquanto que nas sondas de nomeação era seis.

Nas sondas de exclusão, nenhuma criança apresentou acertos no pré-teste. Nas sondagens seguintes, as crianças com DT apresentaram entre dois e cinco acertos para o nome de fruta (S), entre um e dois acertos para a cores das cestas (A) e entre dois e três acertos na cor dos quadrados (A' - exceto nas sondas de manutenção). As crianças com SD, em geral, apresentaram um número menor de acertos, especialmente em relação ao nome de fruta e da cor do quadrado: entre dois e três acertos para $\mathrm{S}$, entre um e dois para $\mathrm{A}$ (como as crianças com DT) e um acerto na Sondagem 1 e dois na Manutenção para A'.

Nas sondas de emparelhamento ao modelo (Figura 2, segundo painel) observa-se que, no pré-teste, nenhuma criança selecionou corretamente a figura do camapu diante da palavra ditada. Entre as crianças do grupo com DT, na primeira sondagem foram verificados sete acertos e, a partir da segunda sondagem, todas as crianças acertaram as duas tentativas com a palavra-alvo. As crianças com SD apresentaram dois acertos em relação ao nome da fruta na segunda sondagem (T2), cinco na terceira (T3) e quatro na Manutenção.

As crianças com DT apresentaram número crescente de acertos em relação à cor (A1 e A1') ao longo das sondagens de emparelhamento ao modelo, com exceção da terceira sondagem (T3) para A1'. As crianças com SD apresentaram números variáveis de acertos ao longo das sondagens em relação à cor. Quando o estímulo era A1, observa-se um desempenho crescente entre a primeira e a segunda sondagem, mas esse desempenho piorou ao longo das sondagens seguintes (de cinco acertos em T2 para três na manutenção). O desempenho das crianças com SD se manteve estável em quatro acertos ao longo de todas as sondagens com o estímulo A1' (exceto na T3, em que não houve nenhum acerto). Observa-se, portanto, que todas as crianças com DT acertaram todas as tentativas de emparelhamento ao modelo da relação nome-fruta (S1) a partir da segunda sessão de leitura, mas que o desempenho dessas crianças foi inferior nas tarefas que envolviam a relação nome de cor. $\mathrm{O}$ desempenho das crianças com SD foi semelhante ao longo de todas as sondagens, tanto nas relações que envolviam o substantivo (S1), quanto nas tentativas que envolviam o nome da cor (A1 e A1') - entre dois e quatro acertos.

Nas sondas de nomeação, observa-se novamente que o desempenho das crianças com DT foi melhor em relação à fruta-alvo $(\mathrm{S} 1)$ do que em relação à 

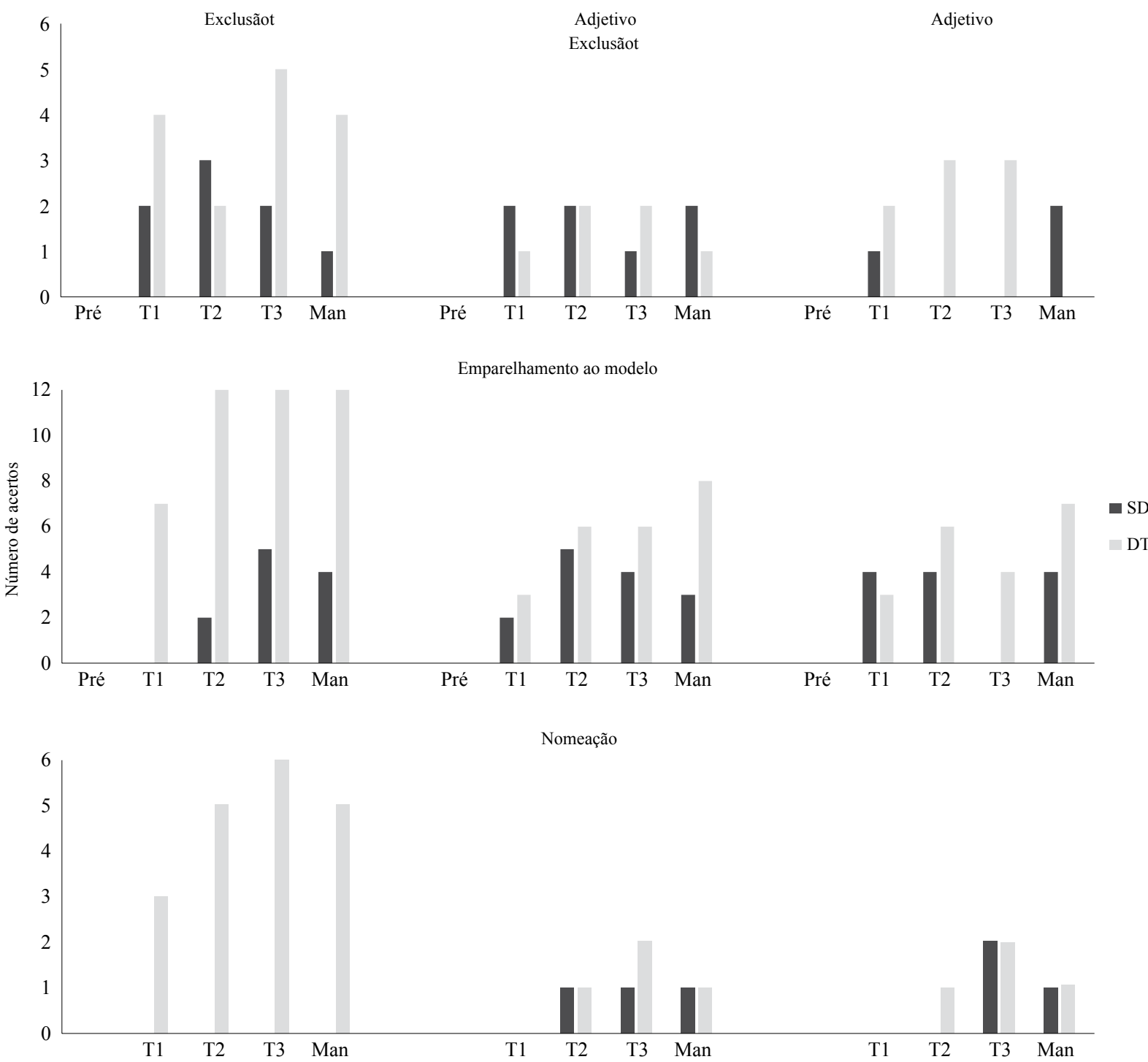

Figura 2. Número de acertos dos grupos (desenvolvimento típico - DT, e Síndrome de Down - SD) nas sondagens (T) realizadas logo após a apresentação de cada condição e uma semana após (Man), nas diferentes tarefas. Os resultados estão separados de acordo com a relação testada: nome-fruta (Substantivo); e nome-cor, apresentada com a figura da cesta (Adjetivo) e com o quadrado colorido (Adjetivo').

cor (A1 e A1'). As crianças com DT apresentaram um número crescente de nomeações corretas de S1 ao longo das sondagens: de três nomeações na primeira sondagem para seis nomeações corretas na Sondagem 3, o que indica que, ao final do procedimento, todas elas nomearam corretamente a figura da fruta. Esse desempenho se manteve na sondagem de Manutenção, exceto para uma criança, que disse não lembrar o nome da fruta.
Nenhuma criança com SD nomeou corretamente o S1, mas algumas pronunciaram vocábulos semelhantes à palavra "camapu". P2, por exemplo, nomeou o estímulo-alvo de "pupú" e "pú" nas duas últimas sondagens; P3, na segunda e terceira sondagens, nomeou S1 de "pupú"; P4 nomeou S1 como "catapú" na segunda sondagem e na Manutenção e como "papú" na terceira; P5, na fase de Manutenção, nomeou S1 de "bapú". 
Em relação à nomeação da cor bege, na primeira sondagem (T1) nenhuma criança com DT ou com SD nomeou corretamente a cor, seja quando ela aparecia na cesta (A1), seja quando apresentada no quadrado (A1'). Nas sondagens subsequentes, verificou-se no máximo duas nomeações corretas em cada grupo (o que corresponde a dois participantes individuais) para A1 ou A1'. No grupo das crianças com DT, quatro dos seis participantes nomearam a cor como "amarelo" (tanto para A1 como para A1'); entre as crianças com SD, o mesmo ocorreu com três crianças.

Os resultados das sondas de generalização não estão representados na Figura 2 por terem sido aferidos apenas na fase de Manutenção. De modo geral, houve um maior número de acertos no desempenho de generalização em relação à fruta do que à cor: cinco crianças com DT selecionaram a fruta correta diante da palavra "camapu", quatro selecionaram a cesta correta diante de "bege" e duas selecionaram corretamente o bloquinho de madeira diante do nome da cor. Entre as crianças com SD, duas selecionaram a fruta correta, uma selecionou a cesta com a cor correspondente e outra criança selecionou o bloquinho de madeira correto.

\section{Discussão}

De modo geral, observou-se que os resultados das crianças com DT foram superiores aos das crianças com SD em todas as medidas realizadas. Além disso, observou-se entre as crianças com DT, um número maior de acertos relacionados à relação nome-fruta, em comparação às relações nome-cor testadas, para todas as medidas realizadas, o que confirma dados de outras pesquisas (e.g., Walsh \& Blewitt, 2006). Os resultados apresentados indicam que as condições de leitura compartilhada realizadas, assim como o arranjo da apresentação dos estímulos visuais nas ilustrações do livro, não foram suficientes para a aprendizagem das relações-alvo pela maioria das crianças com SD, apesar de se ter verificado a aprendizagem da relação nome-fruta entre as crianças com DT.

A aprendizagem de palavras a partir da leitura compartilhada de histórias envolve uma série de repertórios comportamentais. Repertórios de “ouvinte" (Skinner, 1957) são requeridos para discriminar as palavras e figuras "novas" que apareceram na história, de outras palavras e figuras completamente desconhecidas e que não fazem parte do enredo (desempenho de exclusão), e para relacionar as palavras novas às suas respectivas figuras (desempenho de emparelhamento ao modelo) e objetos tridimensionais (generalização). Repertórios de "falante" também devem ocorrer, seja para repetir, de forma aberta ou encoberta, as palavras desconhecidas, para responder perguntas sobre aspectos da história relacionados aos aspectos desconhecidos, ou para dizer o nome da fruta ou da cor diante de sua figura - desempenho de nomeação- (Garcia, Vaz \& Schmidt, 2016). No entanto, a aprendizagem desses diferentes repertórios a partir da leitura compartilhada não parece ser uniforme entre as crianças, como evidenciado pelos resultados aqui descritos, assim como pelos resultados de Garcia et al. (2016).

Os resultados das crianças com DT deste estudo nos diferentes testes de aprendizagem evidenciaram que o conjunto de condições a que as crianças foram expostas, promoveu aumento de desempenhos corretos, especialmente nas tarefas de emparelhamento ao modelo e nomeação para a relação nome-fruta. Um fator que pode ter auxiliado no desempenho acurado das crianças com DT nessa relação foi a apresentação da figura-alvo em configurações semelhantes àquelas utilizadas em procedimentos experimentais de ensino por exclusão, nas quais o estímulo desconhecido aparece entre outros conhecidos (e.g. de Souza \& de Rose, 2006; Dixon, 1977; Ferrari, de Rose \& McIlvane, 2008; Schmidt, Franco, Lotério \& Gomes, 2016). A utilização de apenas um ou dois estímulos como contrastes conhecidos nas fotos que ilustravam o livro foi empregada porque, conforme Horst, Scott 
e Pollard (2010), quando há um número menor de estímulos concorrentes, há maior possibilidade de a criança aprender por exclusão. $\mathrm{O}$ fato de as crianças com DT mostrarem desempenho acurado nas sondas de emparelhamento e de nomeação da relação nome-fruta a partir da segunda sondagem sugere que a configuração das ilustrações pode ter desempenhado um papel fundamental na aprendizagem dessa relação.

Os resultados obtidos com as crianças com DT na relação nome-cor, no entanto, foram inferiores aos obtidos com a relação nome-fruta. Não se pode atribuir essa dificuldade ao número de relações desconhecidas (duas) presentes na história, uma vez que outras pesquisas já demonstraram que crianças da idade das participantes desta pesquisa conseguem aprender duas novas relações palavra-figura presentes em uma história (Garcia et al., 2016; Horst et al., 2011; Williams \& Horst, 2014). Sendo assim, a diferença encontrada pode ser atribuída às características específicas dos estímulos presentes na situação: em uma das relações a palavra estava relacionada a um objeto (fruta) enquanto que na outra, estava relacionada a uma propriedade do objeto (sua cor).

A diferença na aprendizagem de substantivos e adjetivos já foi extensamente relatada pela literatura (e.g. Booth \& Waxman, 2009; Tribushinina et al., 2013), inclusive em estudos sobre a aprendizagem por exclusão (Holland, Simpson \& Riggs, 2015; Ribeiro, Gallano, Hollanda Souza \& de Souza, 2017). A eventual dificuldade de aprender adjetivos (propriedades de objetos) em comparação a substantivos concretos (objetos) pode estar relacionada a distintos aspectos de controle de estímulos envolvidos nos dois tipos de aprendizagem. Aprender adjetivos a partir de uma história requer que a criança fique sob controle de pistas (gramaticais, por exemplo) presentes nas sentenças, que indiquem que a palavra nova se refere a uma característica do objeto (Mintz, 2005) e não ao objeto como um todo, o que torna a tarefa mais complexa do que aprender substan- tivos. As fotos que ilustravam o livro, que apresentavam dois ou três objetos idênticos na forma (cestas), mas que diferiam apenas em um aspecto (a cor), e o uso do contraste de cores conhecidas (preto e branco) junto com a cor desconhecida (bege), poderiam facilitar a aprendizagem do adjetivo (cf. Tribushinina et al., 2013). No entanto, essa configuração não foi suficiente para que o desempenho das crianças com DT fosse semelhante nas relações nome-cor e nome-fruta, tampouco para as crianças com SD.

É preciso ponderar, porém, que a aprendizagem da nova cor pode ter sido dificultada pelo fato dela ser uma extensão do espectro do amarelo, já conhecido de alguns participantes. Algumas crianças, durante as sondas de nomeação, nomearam as figuras coloridas de "bege" como "amarelo". Nesse caso, as crianças podem, durante a história, ter rejeitado a palavra bege como cor uma vez que, ao olhar para o cesto, podem ter relacionado a cor ao amarelo, e relacionado a palavra bege ao objeto como um todo. Os resultados obtidos não permitem que essa hipótese seja confirmada ou não, mas esse tipo de problema pode ser evitado em estudos futuros pelo uso de substantivos e adjetivos inexistentes no vocabulário corrente da língua portuguesa, como palavras inventadas e objetos construídos pelos pesquisadores, com características como forma ou textura também inventadas, como já verificado em outras pesquisas (Ribeiro et al., 2017; Malerbo \& Schmidt, 2017). No presente estudo, a opção por palavras correntes na língua portuguesa deveu-se ao fato de se trabalhar com uma população com dificuldades de linguagem e não nos pareceu ético o ensino de relações auditivo-visuais descontextualizadas do cotidiano das crianças. Essa é uma limitação do trabalho e precisa ser levada em conta na análise dos resultados.

A despeito dos resultados obtidos com crianças com DT, os obtidos com as crianças com SD não indicaram aprendizagem consistente de nenhuma das duas relações ao longo das sondagens. Apenas duas participantes (P2 e P4) mostraram indícios de 
aprendizagem nas sondas de emparelhamento ao modelo da relação palavra-fruta, uma vez que ambas passaram a relacionar corretamente o estímulo visual ao auditivo a partir da terceira sondagem de aprendizagem. Além disso, tanto P2 quanto P4 passaram a nomear a fruta com nomes próximos ao correto (pupú e catapú, respectivamente) a partir da Sondagem 2 (P4) e da Sondagem 3 (P2). Considerando que a maioria dos participantes com DT passou a apresentar respostas corretas nessas tarefas a partir da primeira sondagem, os resultados dos participantes com SD sugerem que talvez eles precisassem de um número maior de exposições às palavras (e à história) para demonstrarem desempenho acurado nas tarefas propostas.

Diversos pesquisadores (Costa, Grisante, Domeniconi, de Rose \& de Souza, 2013; Horst, 2013; Horst \& Samuelson, 2008; Mather \& Plunkett, 2009; McLeod \& McDade, 2011; Schmidt et al., 2016; Wilkinson, Ross \& Diamond, 2003) discutem que o número de exposições é um fator importante para a aprendizagem de palavras, especialmente em situações de ensino incidental, como aquelas em que a criança precisa responder por exclusão, e que um número reduzido de exposições da relação palavra-objeto/figura não garante a aprendizagem. Há diversas variáveis que podem interferir no número necessário de exposições para aprender um vocábulo novo, como a idade das crianças (Schmidt et al., 2016; Wilkinson et al., 2003), a forma de apresentação dos estímulos nessas exposições (Horst et al., 2011; Horst, 2013; McLeod \& McDade, 2011; Williams \& Horst, 2014), a presença ou não de ensino direto (reforçamento diferencial) das novas relações ou a qualidade das experiências da criança com as relações a serem aprendidas, como a apresentação da palavra/figura/objeto em múltiplos contextos, nos quais se requer da criança, diferentes tipos de desempenhos relacionados a esses estímulos (Cahill \& Greer, 2014; Greer \& $\mathrm{Du}, 2015)$. Consequentemente, um dos fatores que pode ter dificultado a aprendizagem das no- vas palavras pelo grupo com DT foi um número insuficiente de exposições, não só em função das características específicas das crianças (como as suas dificuldades na aprendizagem de repertórios verbais), mas também pela ausência de arranjos mais diretos de ensino durante a leitura.

Intervenções bem-sucedidas com crianças com SD utilizando a leitura compartilhada de histórias (Burgoyne et al., 2012; van Bysterveldt et al., 2006) em geral focalizam habilidades de consciência fonológica, mas, para tal, empregam procedimentos de longa duração (sessões diárias ao longo de seis ou até de 20 semanas). É possível, portanto, que a reexposição das crianças com SD à história pudesse promover as aprendizagens testadas neste estudo. Para além do número de exposições, porém, é importante considerar outras variáveis que podem ter dificultado a aprendizagem das crianças com SD.

Para a realização das tarefas apresentadas nas sondagens (exclusão, emparelhamento ao modelo, nomeação e generalização) era necessário que a criança apresentasse um conjunto de desempenhos de falante e de ouvinte (Greer \& Du, 2015; Skinner, 1957), o que, em conjunto, evidenciaria a aprendizagem da palavra. Para que isso ocorresse, porém, era necessário que durante as leituras as crianças ouvissem a história e inspecionassem as ilustrações apresentadas, relacionando os elementos desconhecidos nessas ilustrações às palavras também desconhecidas. Além disso, a exposição à leitura e às ilustrações deveria ter funcionado como uma espécie de "linha de base" para que elas identificassem os estímulos novos $\mathrm{S} 1$ e A1/ A1' como estímulos "familiares" em alguma medida, em contraste com estímulos completamente conhecidos (e.g., uma maçã) ou completamente desconhecidos (e.g., a fruta pitaia). A experiência de ecoar a palavra-alvo ou de responder a uma pergunta cuja resposta fosse a palavra-alvo (tato intraverbal) também deveria contribuir para os desempenhos requeridos nos testes. Finalmente, se as figuras exercessem controle sobre diferentes 
repertórios da criança, tal controle deveria se estender para os objetos em si. Todos esses desempenhos se constituíam em repertórios necessários para a atividade e talvez não tivessem sido aprendidos em outras oportunidades pela criança, de tal forma que, quando exposta à leitura da história, não tivesse ainda o repertório requerido para aprender as novas relações apenas a partir das sessões de leitura. Nesse sentido, ainda que as condições de leitura programadas para este estudo (a repetição da mesma história, a emissão de ecoico durante a leitura e a exigência de responder perguntas relacionadas à palavra-alvo) pudesse favorecer os processos acima descritos, foram insuficientes para as crianças estudadas.

Para que a situação de ensino incidental de palavras por meio da leitura de histórias seja efetiva para crianças com SD (ou com algum tipo de atraso no desenvolvimento), é necessário que se investigue as condições críticas que devem estar presentes no repertório desses indivíduos. Futuras pesquisas, portanto, deveriam focalizar essa questão, investigando o repertório de entrada necessário para que a aprendizagem de vocabulário ocorra na situação de leitura compartilhada, mas também testando atividades complementares à leitura de histórias que podem fornecer às crianças as experiências necessárias para a aprendizagem.

No presente estudo foram investigadas atividades simples, como a repetição das palavras-alvo durante a leitura e a resposta a perguntas que exigiam a emissão das palavras-alvo, atividades indicadas por outras pesquisas como favorecedoras da aprendizagem (Blewitt \& Langan, 2016). Pesquisas que testam programas de intervenção com leitura de histórias, por outro lado, sugerem muitas outras atividades, que proporcionam às crianças experiências múltiplas com as palavras novas e exigem variados desempenhos com elas (e.g., Lever \& Sénéchal, 2011; Pollard-Durodola et al., 2011). O estudo sobre os repertórios necessários para a aprendizagem incidental a partir da leitura compartilhada de histórias pode indicar procedimentos específicos a serem testados em futuras intervenções para crianças com atrasos no desenvolvimento.

\section{Referências}

Andrade, C. R. F., Befi-Lopes, D. M., Fernandes, F. D. M., \& Wertzner, H. F. (2004). ABFW-Teste de linguagem infantil nas áreas de fonologia, vocabulário, fluência e pragmática. Barueri: Pró Fono.

Blewitt, P., \& Langan, R. (2016). Learning words during shared book reading: the role of extratextual talk designed to increase child engagement. Journal of Experimental Child Psychology, 150, 404-410. Doi: 10.1016/j.jecp.2016.06.009

Booth, A. E., \& Waxman, S. R. (2009). A horse of a different color: Specifying with precision infants' mappings of novel nouns and adjectives. Child Development, 80(1), 15-22. Doi: 10.1111/j.1467-8624.2008.01242.x

Burgoyne, K., Duff, F. J., Clarke, P. J., Buckley, S., Snowling, M. J., \& Hulme, C. (2012). Efficacy of a reading and language intervention for children with down syndrome: a randomized controlled trial. Journal of Child Psychology and Psychiatry, 53(10), 1044-1053. Doi: 10.1111/j.1469-7610.2012.02557.x

Cahill, C. S., \& Greer, R. D. (2014). Actions vs. words: how we can learn both. Acta de Investigación Psicológica, 4(3), 1717-1746. Doi: 10.1016/S2007-4719(14)70976-7

Chow, B. W., McBride-Chang, C., Cheung, H., \& Chow, C. S. (2008) Dialogic reading and morphology training in Chinese children: Effects on language and literacy. Developmental Psychology, 44(1), 233-244. Doi: 10.1037/00121649.44.1.233

Costa, A. R. A., Gristante, P. C., Domeniconi, C., de Rose, J. C. C., \& de Souza, D. G. (2013). Naming new stimuli after selection by exclusion. Paidéia, 23(55), 217-224. Doi: 10.1590/198243272355201309 
de Souza, D. G., \& de Rose, J. C. (2006). Desenvolvendo programas individualizados para o ensino de leitura. Acta Comportamentalia, 14(1), 77-98.

Dixon, L. S. (1977). The nature of control by spoken words over visual stimulus selection. Journal of the Experimental Analysis of Behavior, 27(3), 433- 442. Doi: 10.1901/jeab.1977.27-433

Evans, M. A., \& Saint-Aubin, J. (2013). Vocabulary acquisition without adult explanations in repeated shared book reading: an eye movement study. Journal of Educational Psychology, 105(3), 596-608. Doi: 10.1037/a0032465

Ferrari, C., de Rose, J. C., \& McIlvane, W. J. (2008). A comparison of exclusion and trial-and-error procedures: primary and secondary effects. Experimental Analysis of Human Behavior Bulletin, 29, 9-16.

Freire, R. C. L., Duarte, N. S., \& Hazin, I. (2012). Fenótipo neuropsicológico de crianças com Síndrome de Down. Psicologia em Revista, 18(3), 354-372. Doi: 10.5752/P.16789563.2012v18n3p354

Garcia, F. P., Vaz, A. M., \& Schmidt, A. (2016). Shared book reading and word learning in preschool children. Temas em Psicologia, 24(4), 14371449. Doi: 10.9788/TP2016.4-14En

Gentner, D. (1982). Why nouns are learned before verbs: linguistic relativity versus natural partitioning. Center for the Study of Reading Technical Report; no. 257.

Greer, R. D., \& Du, L. (2015). Experience and the onset of the capability to learn names incidentally by exclusion. The Psychological Record, 65, 355-373. Doi: 10.1007/s40732-014-0111-2

Greer, R. D., \& Longano, J. (2010). A rose by naming: how we may learn how to do it. The Analysis of Verbal Behavior, 26(1), 73-106. Doi: 10.1007/ BF03393085

Greer, R. D., \& Ross, D. E. (2008). Verbal behavior analysis: Inducing and expanding complex communication in children with severe language delays. Boston: Allyn \& Bacon;
Hargrave, A. C., \& Sénéchal, M. (2000). A book reading intervention with preschool children who have limited vocabularies: the benefits of regular reading and dialogic reading. Early Childhood Research Quarterly, 15(1), 75-90. Doi: 10.1016/S0885-2006(99)00038-1

Holland, A., Simpson, A., \& Riggs, K. J. (2015). Young children retain fast mapped object labels better than shape, color, and texture words. Journal of Experimental Child Psychology, 134, 1-11. Doi: 10.1016/j.jecp.2015.01.014

Horst, J. S. (2013). Context and repetition in word learning. Frontiers in Psychology, 1- 30. Doi: 10.3389/fpsyg.2013.00149

Horst, J. S., Parsons, K. L., \& Bryan, N. M. (2011). Get the story straight: contextual repetition promotes word learning from storybooks. Frontiers in Developmental Psychology, 2(17), 1-11. Doi: 10.3389/fpsyg.2011.00017

Horst, J. S., \& Samuelson, L. K. (2008). Fast mapping but poor retention by 24-month-old infants. Infancy, 13(2), 128-157.

Horst, J. S., Scott, E. J., \& Pollard, J. A. (2010). The role of competition in word learning via referent selection. Developmental Science, 13(5), 706713. Doi: 10.1111/j.1467-7687.2009.00926.x Kaefer, T., Pinkham, A. M., \& Neuman, S. B. (2017). Seeing and knowing: attention to illustrations during storybook reading and narrative comprehension in 2-year-olds. Infant and Child Development, 26(5), e2018. Doi: 10.1002/icd.2018

Kucker, S. C., Murray, B., \& Samuelson, L. K. (2015). Slowing down fast mapping: redefining the dynamics of word learning. Child Development Perspectives, 9(2), 74-78. Doi: 10.1111/ cdep. 12110

Lever, R., \& Sénéchal, M. (2011). Discussing stories: on how a dialogic reading intervention improves kindergartners' oral narrative construction. Journal of Experimental Child Psychology, 108(1), 1-24. Doi: 10.1016/j.jecp.2010.07.002

Malerbo, A. C., \& Schmidt, A. (2017). Aprendizagem de relações nome-textura por meio de dois 
procedimentos de ensino por exclusão. Acta Comportamentalia, 25(1), 5-23.

Mather, E., \& Plunkett, K. (2009). Learning words over time: the role of stimulus repetition in mutual exclusivity. Infancy, 14(1), 60-76. Doi: $10.1080 / 15250000802569702$

McLeod, A. N., \& McDade, H. L. (2011). Preschoolers' incidental learning of novel words during storybook reading. Clinical Research in Preschool, 32(4), 256-266. Doi: 10.1177/1525740109354777

Mintz, T. H. (2005). Linguistic and conceptual influences on adjective acquisition in 24- and 36-month-olds. Developmental Psychology, 41, 17-29. Doi: 10.1037/0012-1649.41.1.17

Pollard-Durodola, S. D., Gonzalez, J. E., Simmons, D. C., Kwok, O., Taylor, A. B., Davis, M. J., Kim, M., \& Simmons, L. (2011). The effects of an intensive shared book-reading intervention for preschool children at risk for vocabulary delay. Exceptional Children, 77(2), 161-183. Doi: 10.1177/001440291107700202

Ribeiro, T. A., Gallano, T. P., Hollanda Souza, D., $\&$ de Souza, D. D. G. (2017). Responding and learning by exclusion in 2-year-olds: the case of adjectives. The Psychological Record, 67(3), 293-314. Doi: 10.1007/s40732-016-0213-0

Rogoski, B., Flores, E., Gauche, G., Coêlho, R., \& de Souza, C. (2017). Compreensão após leitura dialógica: efeitos de dicas, sondas e reforçamento diferencial baseados em funções narrativas. Perspectivas em Análise do Comportamento, 6(1), 48-59. Doi: 10.18761/pac.2015.6.1.a04

Schmidt, A., Costa, A. R. A., Norberto, M. C., \& Voss, A. (2014). Ocorrência de classes gramaticais na fala espontânea de crianças de 18 a 41 meses com pares. Psicologia: Reflexão \& Critica, 27(3), 573-581. Doi: 10.1590/16787153.201427319

Schmidt, A., Franco, M. G. O., Lotério, L. S., \& Gomes, G. F. (2016). Learning name-object relations after a single exclusion trial in 18- to 48- month- old children. The Psychological
Record, 66(1), 53-63. Doi: 10.1007/s40732015-0151-2

Sim, S., \& Berthelsen, D. (2014). Shared book reading by parents with young children: Evidence-based practice. Australasian Journal of Early Childhood, 39(1), 50-55.

Skinner, B. F. (1957). Verbal behavior. New York: Appleton-Century-Crofts.

Tardif, T., Gelman, S. A., \& Xu, F. (1999). Putting the "noun bias" in context: A comparison of English and Mandarin. Child Development, 70(3), 620-635. Doi: 10.1111/1467-8624.00045

Tribushinina, E., Bergh, B., Kilani-Schoch, M., Aksu-Koç, A., Dabasinkiené, I., Hrzica, G. et al. (2013). The role of explicit contrast in adjective acquisition: a crosslinguistic longitudinal study of adjective production in spontaneous child speech and parental input. First Language, 33(6), 594- 616. Doi : 10.1177/0142723713503146

Tribushinina, E., \& Gillis, S. (2012). The acquisition of scalar structures: Production of adjectives and degree markers by Dutch-speaking children and their caregivers. Linguistics, 50, 241-268. Doi: 10.1515/ling-2012-0009

van Bysterveldt, A. K., Gillon, G. T., \& Moran, C. (2006). Enhancing phonological awareness and letter knowledge in preschool children with down syndrome. International Journal of Disability, Development and Education, 53(3), 301329. Doi: 10.1080/10349120600847706

Walsh, B. A., \& Blewitt, P. (2006) The effect of questioning style during storybook reading on novel vocabulary acquisition of preschoolers. Early Childhood Education Journal, 33(4), 273- 278. Doi: 10.1007/s10643-005-0052-0

Waxman, S. R., \& Booth, A. E. (2001). Seeing pink elephants: fourteen-month-olds' interpretations of novel nouns and adjectives. Cognitive Psychology, 43, 217-242. Doi: 10.1006/ cogp.2001.0764

Wilkinson, K. M., Ross, E., \& Diamond, A. (2003). Fast mapping of multiple words: insights into when "the information provided" does and does 


\section{Camila Bonagamba, Andréia Schmidt}

not equal "the information perceived". Applied Developmental Psychology, 24, 739-762. Doi: 10.1016/j.appdev.2003.09.006

Williams, E. S., \& Horst, J. S. (2014). Goodnight book: Sleep consolidation improves word learning via storybooks. Frontiers in Psychology, 5, 1-12. Doi: 10.3389/fpsyg.2014.00184

\section{Recebido: 29 de julho de 2017}

Aprovado: 08 de junho de 2018 